Inpatient Deaths of Patient with Cardiac Devices

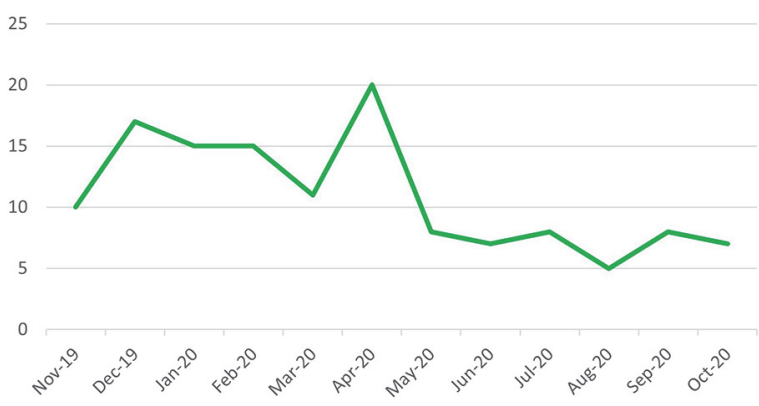

Abstract 109 Figure 1

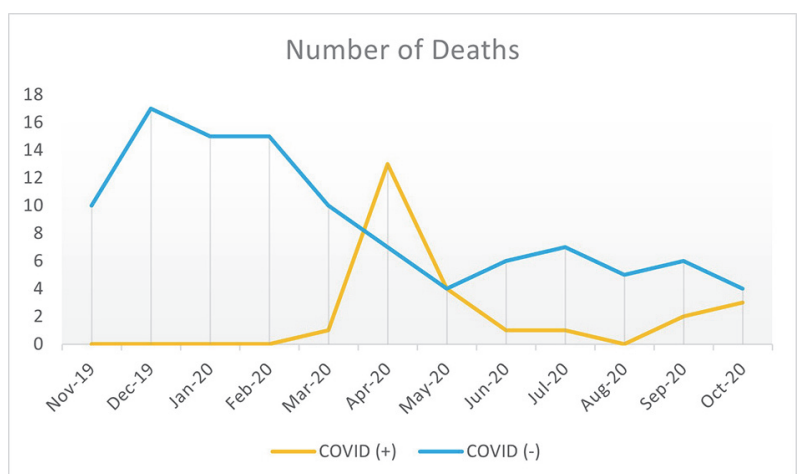

Abstract 109 Figure 2

identified and their COVID-19 infection status determined from the death certificate.

Results 131 in-patients (76\% male) with implantable cardiac devices (64\% pacemakers, 23\% biventricular pacemakers, and $13 \%$ defibrillators) died during the study period. Mean age of death was 84 years (SD \pm 8 years). Most deaths (91\%) occurred on the medical ward (including level 2 respiratory care unit). There were more deaths from March 2020, peaking in April 2020, before gradually declining in May 2020, correlating with the first wave of the COVID-19 pandemic in the UK (figure 1). The majority of patients who died in April 2020 were COVID positive. From early September 2020 onwards, the number of COVID positive deaths started to increase again, correlating with the second wave. Following the declaration of the pandemic, deaths occurred mostly amongst COVID negative patients (figure 2).

Conclusion Inpatient mortality of patients with cardiac device were highest in the elderly population which predominantly occurred during the first wave of COVID-19 pandemic.

Conflict of Interest none

\section{SYSTEMATIC REVIEW OF SAFETY AND EFFICACY OF RENAL SYMPATHETIC DENERVATION FOR THE MANAGEMENT OF CARDIAC ARRHYTHMIAS}

Nakulan Nantha Kumar. University of Bristol, Bristol, UK

10.1136/heartjnl-2021-BCS.109

Background Renal sympathetic denervation (RSD) has an emerging role in treating cardiac arrythmias, especially Atrial
Fibrillation (AF), as reported by several studies and due to its role in reducing blood pressure and speculation on its effects on the autonomic nervous system. In the wake of the controversy surrounding the SMYPLICITY HTN-3 trial and data from subsequent trials, this review aims to perform an updated and more comprehensive review of the impact of RSD on atrial and ventricular arrhythmias. Method: A systematic search included the Medline, Scopus and Embase databases using the terms 'Renal Denervation' AND 'Arrhythmias or Atrial or Ventricular', limited to Human and English language studies within the last 10 years. This search yielded, 19 relevant studies $(n=6$ randomised controlled trials, $n=13$ nonrandomised cohort studies) which comprised 783 patients.

Results Upon analysis, the data shows RSD is a safe procedure and is not associated with increases in complications or mortality post procedure. Importantly, there is no evidence RSD is associated with a deterioration in renal function, even in patients with chronic kidney disease. RSD with or without adjunctive Pulmonary Vein Isolation (PVI) was associated with improvements in freedom from AF, premature atrial complexes (PACs), Ventricular arrythmias and other Echocardiographic parameters. Significant reductions in both ambulatory and office blood pressure were also observed in the majority of studies.

Conclusion The findings of this review support the findings of so called 'second generation' RSD studies which report that RSD is safe and is associated with reductions in short term blood pressure, AF burden and key Echocardiological indices like LVEF. However, there is a paucity of evidence regarding the impact of RSD on other less prominent arrythmia subtypes. Hence, large standardized multi-centre RCTs investigating the role of RSD are necessary to comprehensively assess the efficacy of the procedure in treating all arrythmias. (300 words)

Conflict of Interest N/A

\section{A TERTIARY CENTRE EXPERIENCE COMPARING NEW TEMPERATURE-CONTROLLED HIGH-POWER SHORT- DURATION ABLATION TECHNOLOGY WITH STANDARD RADIOFREQUENCY AND CRYO-ENERGY ABLATION}

${ }^{1}$ Akash Mavilakandy, ${ }^{1}$ Bharat Sidhu, ${ }^{2}$ Sharon Man, ${ }^{1}$ Ibrahim Antoun, ${ }^{1}$ Zakarriya Vali, ${ }^{1}$ Tiago Paggi De Almeida, ${ }^{1}$ Xin Li, ${ }^{2}$ Merzaka Lazdam, ${ }^{2}$ Mokhtar Ibrahim, ${ }^{2}$ ShuiHao Chin, ${ }^{2}$ Alastair Sandilands, ${ }^{2}$ Peter Stafford, ${ }^{2}$ Riaz Somani, ${ }^{1}$ Andre Ng. ${ }^{1}$ University of Leicester, Leicester, UK; ${ }^{2}$ University Hospitals of Leicester

\subsection{6/heartjnl-2021-BCS.110}

Introduction Pulmonary vein isolation (PVI) is an established treatment strategy for atrial fibrillation (AF). Radiofrequency (RF) ablation technology has evolved over the last decade with the very high-power short-duration (vHPSD) temperature-controlled ablation approach emerging as the latest development. The aim of this study was to determine the procedural efficacy, metrics and safety of vHPSD ablation with conventional power-controlled RF (PCRF) ablation and cryoenergy ablation (CRYO).

Methods A prospective single tertiary centre analysis was performed on patients undergoing first time PVI ablation from 2019 to 2020. The population was divided in to 4 treatment arms; QMode Plus (vHPSD), Qmode (Qmode), PCRF using THERMOCOOL SMARTTOUCH @ SF Catheter (STSF) and CRYO. Demographics, clinical and procedural metrics such as PVI duration, ablation time and sedation requirement were 